\title{
Therapeutic potential of vascular stem cells for anterior cruciate ligament reconstruction
}

\author{
Tomoyuki Matsumoto, Koji Takayama, Shinya Hayashi, Takahiro Niikura, Takehiko Matsushita, \\ Ryosuke Kuroda
}

Department of Orthopaedic Surgery, Kobe University Graduate School of Medicine, Kobe, Japan

Correspondence to: Tomoyuki Matsumoto, MD. Department of Orthopaedic Surgery, Kobe University Graduate School of Medicine, 7-5-1, Kusunokicho, Chuo-ku, Kobe 650-0017, Japan. Email: matsun@m4.dion.ne.jp.

Provenance: This is an invited article commissioned by the Section Editor Dr. Yu Zhang (Department of Sport Medicine and Adult Reconstructive Surgery, Drum Tower Hospital, School of Medicine, Nanjing University, China).

Comment on: Sun Y, Chen W, Hao Y, et al. Stem cell-conditioned medium promotes graft remodeling of midsubstance and intratunnel incorporation after anterior cruciate ligament reconstruction in a rat model. Am J Sports Med 2019;47:2327-37.

Submitted Sep 27, 2019. Accepted for publication Nov 13, 2019.

doi: $10.21037 / \mathrm{atm} .2019 .11 .79$

View this article at: http://dx.doi.org/10.21037/atm.2019.11.79

Anterior cruciate ligament (ACL) is well known to have poor potential healing when injured. Most surgical procedures for ACL reconstruction (ACLR) using hamstring tendon grafts require tendon-bone healing and graft maturation, however, the tendon-bone attachment is the weakest area in the surgically created bone tunnel in the early post-operative period. Therefore, acquiring secure tendon-bone integration is an essential task for earlier rehabilitation program and earlier return to work and sports. To enhance tendon-bone healing, tissue engineering techniques with growth factors/cytokines or stem cells have received much attention. Considering enhancement of tendon-bone healing in ACLR, several animal studies have been reported using periosteum, bone marrow mesenchymal stem cells (MSCs) or bone marrow stromal cells $(1,2)$, and growth factors. Recently, similar to growth factors, stem cell conditioned medium (CM) injection was reported to promote graft maturation and tendon-bone integration following ACLR in a rat ACLR model (3). They concluded that human bone marrow stem cell (hBMSC)-CM accelerated tendon-bone incorporation and mid-substance graft maturation, and furthermore confirmed the enhanced proliferation, differentiation, and collagen synthesis of fibroblasts by the in vitro assay. As one of the mechanisms enhancing healing potential, they confirmed up-regulated cytokine including transforming growth factor b1 (TGFb1), vascular endothelial growth factor (VEGF) and insulin growth factor-1 (IGF-1) in the in vitro assay. The effect of $\mathrm{CM}$ has been reported to secrete several cytokines for accelerating healing process and/or controlling inflammation in the field of regenerative medicine. For example, up-regulated effects by $\mathrm{CM}$ in human tenocyte differentiation (4) and anti-inflammatory effects by CM in human synovium and cartilage (5) have been reported. They also suggested that the paracrine effect is one of the key mechanisms for contributing to healing process by inhibiting inflammation and accelerating regenerative tissues. One of the most important advantages of CM may be proper concentration of cytokines necessary for healing in the synovial fluid, in which several cytokines are secreted. Whereas these biological approaches including CM for ACLR are currently experimental, surgeons expect their therapeutic feasibility in clinical settings in the near future.

Over the last decade, surgeons have considered ACL intrinsic healing potential as a controversy. Almost all surgeons recognize that ACL rupture needs reconstruction, in which vascular supply and intrinsic cell migration are insufficient. On the other hand, some surgeons have reported that ACL spontaneously heals only with primary suture (6). In fact, during arthroscopic procedures for ACLR in acute stage, a tibial stump is often connecting fibers to the wall of femur or posterior cruciate ligament, suggesting that ACL has potential healing. However, exact 
evidence proofing ACL healing potential is lacking. Stem cells with high proliferation and multi-differentiation potential are one of the reasonable explanations for ACL potential healing. Although several scientific reports indicate that MSC-like cells exist in human ACL tissues $(7,8)$, further detailed studies are necessary for exploring the cell characteristic feature and the origin. Blood vessels have been recognized to supply stem/progenitor cells with a characteristic of CD34 and CD146 surface marker (9). Matsumoto et al. demonstrated that CD34-positive vascular cells are recruited in sub-acutely ruptured ACL tissues and confirmed that these cells had high proliferation and multidifferentiation potential in the in vitro experiment (10). They also confirmed that the vascularity is richer in the ruptured site than in the mid-substance region using immunohistochemical vascular staining. In addition, CD34-positive and CD146-positive cells in the ACL ruptured site were confirmed by flow cytometry and immunohistochemistry. These cells were confirmed to have the capacity for multilineage differentiation including osteogenic, adipogenic, chondrogenic, and endotheliogenic differentiation. As the next series, Mifune et al. reported that intra-capsular administration of human ACL-derived vascular CD34-positive cells accelerated tendon-bone healing by enhancing angiogenesis/vasculogenesis and osteogenesis in the surgically created bone tunnels using an immunodeficient rat ACLR model (11). Molecular approach demonstrated that intrinsic angiogenesis and osteogenesis and human-derived endothelial differentiation (vasculogenesis) and osteogenic differentiation were enhanced by intra-capsular transplantation of human ACL-derived vascular CD34-possiteive cells. In addition, biomechanical testing as well as histological and radiological findings confirmed early tendon-bone healing potential by vascular CD34-possiteive cell transplantation in the animal model. It was of note that human non-selected cells as well as CD34-positive cells contributed to tendon-bone healing and reduction of tunnel enlargement in the animal model. When cell therapy is applied to ACLR, cell transplantation needs second-step arthroscopic surgery for cell isolation and cell expansion. Based on the evidence that ACL ruptured site has rich supply of CD34-positive cells (10) and human non-selected cells as well as CD34-positive sorted cells were useful in xenotransplantation model (11), Matsumoto et al. aimed to test the therapeutic potential of ACL ruptured tissue, not CD34-positive sorted cells, using an autologous transplantation ACLR model in a large animal (12). In this study, they harvested ACL ruptured tissue two days after
ACL resection and sutured to the tibial graft in the tibial tunnel in ACLR. Early tendon-bone healing and reduction of tunnel enlargement were confirmed by biomechanical testing as well as histology and computed-tomography (CT) when compared with no tissue control group. These pre-clinical studies could demonstrate the effectiveness of ruptured tissue for ACLR in clinical settings.

Given this background, as a pilot study, Matsumoto et al. performed double-bundle (DB) ACLR with and without the autologous ruptured tissue suturing to the grafts in patients with sub-acute ACL injury to compare femoral/ tibial tunnel enlargement and two-year clinical outcomes (13). In the study, ten patients with sub-acute ACL rupture were randomly selected to perform DB ACLR with the ruptured tissue suturing to femoral/tibial hamstring grafts or conventional DB ACLR as a control group. The results exhibited significant reduction of tunnel enlargement evaluated by 3 dimensional-multidetector CT in the group that underwent DB ACLR with suturing, especially at the femoral side. However, the post-operative clinical outcomes including anterior knee stability assessed with the KT-1000 arthrometer, rate of pivot shift test, and Lysholm score showed no significant difference between the two groups. Among several reports applying cell therapy or regenerative medicine for ACLR, only periosteum was applied for human ACLR. Chen et al. reported satisfactory results with the periosteum-enveloping hamstring tendon graft in single-bundle ACLR with minimal tunnel widening in the 2-7 years clinical follow-up of 312 patients. Compared with the study done by Matsumoto et al., the concept of the strategy was similar and tunnel reduction was confirmed by X-ray assessment (14). If the treatment using ACL ruptured tissue has advantages over previous treatments for enhancing tendon-bone healing, the ruptured ACL tissue can be easily used in clinical settings as it does not need cell isolation and expansion, additional incision and procedure, and 2-step arthroscopic surgery.

To predict outcomes of ACLR, the characteristics of patients may be important. Age is well known to affect healing potential of cells. Uefuji et al. (15) found that ACL ruptured tissues from teenagers contained more CD34-positive cells compared with those from adults over 30-year-old. Furthermore, cells from adolescents displayed a higher proliferation and multilineage differentiation potential in the in vitro assay. Consistent with the finding, Nakano et al. (16) confirmed that ACL-derived vascular cells harvested from adolescents were superior to those of adults over 30-year-old in their capacity enhancing tendon- 
bone healing in an immunodeficient rat model of ACLR. The in vitro study (17) also confirmed that ruptured ACL tissues harvested within 3 months of rupture had more CD34-positive cells and exhibited an enhanced potential for osteogenesis and angiogenesis. The time taken from ACL injury to surgery was also previously reported to affect healing potential of ACL remnant cells. Naraoka et al. reported that gene expression patterns of ACL remnant cells were time-dependently altered after ACL injury (18), and Nohmi et al. reported the declined potential of ACL remnant cells for colony formation and differentiation over time after ACL injury (19). Lee et al. reported that freshly isolated MSCs were more found in ACL-derived cells from patients with subacute tear compared with those with chronic ACL injury (20). Moreover, Zhang et al. reported that a higher proliferation and multilineage differentiation potential was found in ACL remnant cells when the time taken from injury to surgery was short (17). Inokuchi et al. showed a greater tendon-bone healing potential with human ACL remnant cells obtained from early postinjury phase in an immunodeficient rat ACLR model than cell transplantation obtained from chronic phase (21). Furthermore, Kirizuki et al. reported that the nonreattachment remnants, compared with the reattachment remnants, have more CD34-positive cells in ACL ruptured tissues and exhibited higher potential for proliferation and multilineage differentiation, especially in osteogenic and endotheliogenic differentiation (22). Considering these findings, when surgeons perform remnant preserving ACLR or ACLR with ruptured tissue, the patient's age, time taken from ACL injury to surgery, and remnant types should also be considered when predicting healing ability. In the future, other demographic factors including gender, type of injury, patient activity level, and characteristics of ACL derived cells for the healing potential should be assessed.

\section{Acknowledgments}

None.

\section{Footnote}

Conflicts of Interest: The authors have no conflicts of interest to declare.

Ethical Statement: The authors are accountable for all aspects of the work in ensuring that questions related to the accuracy or integrity of any part of the work are appropriately investigated and resolved.

\section{References}

1. Lim JK, Hui J, Li L, et al. Enhancement of tendon graft osteointegration using mesenchymal stem cells in a rabbit model of anterior cruciate ligament reconstruction. Arthroscopy 2004;20:899-910.

2. Karaoglu S, Celik C, Korkusuz P. The effects of bone marrow or periosteum on tendon-to-bone tunnel healing in a rabbit model. Knee Surg Sports Traumatol Arthrosc 2009;17:170-8.

3. Sun Y, Chen W, Hao Y, et al. Stem Cell-Conditioned Medium Promotes Graft Remodeling of Midsubstance and Intratunnel Incorporation After Anterior Cruciate Ligament Reconstruction in a Rat Model. Am J Sports Med 2019;47:2327-37.

4. Ekwueme EC, Shah JV, Mohiuddin M, et al. Cross-Talk Between Human Tenocytes and Bone Marrow Stromal Cells Potentiates Extracellular Matrix Remodeling In Vitro. J Cell Biochem 2016;117:684-93.

5. van Buul GM, Villafuertes E, Bos PK, et al. Mesenchymal stem cells secrete factors that inhibit inflammatory processes in short-term osteoarthritic synovium and cartilage explant culture. Osteoarthritis Cartilage 2012;20:1186-96.

6. Marshall JL, Warren RF, Wickiewicz TL, et al. The anterior cruciate ligament: a technique of repair and reconstruction. Clin Orthop Relat Res 1979:97-106.

7. Lee IC, Wang JH, Lee YT, et al. Development of a useful technique to discriminate anterior cruciate ligament cells and mesenchymal stem cells--the application of cell electrophoresis. J Biomed Mater Res A 2007;82:230-7.

8. Lee SY, Miwa M, Sakai Y, et al. In vitro multipotentiality and characterization of human unfractured traumatic hemarthrosis-derived progenitor cells: A potential cell source for tissue repair. J Cell Physiol 2007;210:561-6.

9. Crisan M, Yap S, Casteilla L, et al. A perivascular origin for mesenchymal stem cells in multiple human organs. Cell Stem Cell 2008;3:301-13.

10. Matsumoto T, Ingham SM, Mifune $Y$, et al. Isolation and characterization of human anterior cruciate ligament-derived vascular stem cells. Stem Cells Dev 2012;21:859-72.

11. Mifune $\mathrm{Y}$, Matsumoto T, Ota S, et al. Therapeutic potential of anterior cruciate ligament-derived stem cells for anterior cruciate ligament reconstruction. Cell Transplant 2012;21:1651-65. 
12. Matsumoto T, Kubo S, Sasaki K, et al. Acceleration of tendon-bone healing of anterior cruciate ligament graft using autologous ruptured tissue. Am J Sports Med 2012;40:1296-302.

13. Matsumoto T, Kuroda R, Matsushita T, et al. Reduction of tunnel enlargement with use of autologous ruptured tissue in anterior cruciate ligament reconstruction: a pilot clinical trial. Arthroscopy 2014;30:468-74.

14. Chen CH, Chang CH, Su CI, et al. Arthroscopic singlebundle anterior cruciate ligament reconstruction with periosteum-enveloping hamstring tendon graft: clinical outcome at 2 to 7 years. Arthroscopy 2010;26:907-17.

15. Uefuji A, Matsumoto T, Matsushita T, et al. AgeRelated Differences in Anterior Cruciate Ligament Remnant Vascular-Derived Cells. Am J Sports Med 2014;42:1478-86.

16. Nakano N, Matsumoto T, Takayama K, et al. Agedependent healing potential of anterior cruciate ligament remnant-derived cells. Am J Sports Med 2015;43:700-8.

17. Zhang S, Matsumoto T, Uefuji A, et al. Anterior cruciate ligament remnant tissue harvested within 3-months after injury predicts higher healing potential. BMC

Cite this article as: Matsumoto T, Takayama K, Hayashi S, Niikura T, Matsushita T, Kuroda R. Therapeutic potential of vascular stem cells for anterior cruciate ligament reconstruction. Ann Transl Med 2019;7(Suppl 8):S286. doi: 10.21037/ atm.2019.11.79
Musculoskelet Disord 2015;16:390.

18. Naraoka T, Ishibashi Y, Tsuda E, et al. Time-dependent gene expression and immunohistochemical analysis of the injured anterior cruciate ligament. Bone Joint Res 2012;1:238-44.

19. Nohmi S, Yamamoto Y, Mizukami H, et al. Post injury changes in the properties of mesenchymal stem cells derived from human anterior cruciate ligaments. Int Orthop 2012;36:1515-22.

20. Lee DH, Ng J, Chung JW, et al. Impact of chronicity of injury on the proportion of mesenchymal stromal cells derived from anterior cruciate ligaments. Cytotherapy 2014;16:586-98.

21. Inokuchi T, Matsumoto T, Takayama K, et al. Influence of the Injury-to-Surgery Interval on the Healing Potential of Human Anterior Cruciate Ligament-Derived Cells. Am J Sports Med 2017;45:1359-69.

22. Kirizuki S, Matsumoto T, Ueha T, et al. The Influence of Ruptured Scar Pattern on the Healing Potential of Anterior Cruciate Ligament Remnant Cells. Am J Sports Med 2018;46:1382-8. 